PROCEEDINGS OF THE

AMERICAN MATHEMATICAL SOCIETY

Volume 130, Number 2, Pages 533-542

S 0002-9939(01)06115-9

Article electronically published on June 6, 2001

\title{
GOOD AND VISCOSITY SOLUTIONS OF FULLY NONLINEAR ELLIPTIC EQUATIONS
}

\author{
ROBERT JENSEN, MACIEJ KOCAN, AND ANDRZEJ ŚWIĘCH \\ (Communicated by David S. Tartakoff)
}

\begin{abstract}
We introduce the notion of a "good" solution of a fully nonlinear uniformly elliptic equation. It is proven that "good" solutions are equivalent to $L^{p}$-viscosity solutions of such equations. The main contribution of the paper is an explicit construction of elliptic equations with strong solutions that approximate any given fully nonlinear uniformly elliptic equation and its $L^{p}$-viscosity solution. The results also extend some results about "good" solutions of linear equations.
\end{abstract}

\section{INTRODUCTION}

We are interested in nonlinear elliptic partial differential equations of the form

$$
G\left(x, u(x), D u(x), D^{2} u(x)\right)=0 \quad \text { for } x \in \Omega .
$$

Here

$$
G: \Omega \times \mathbb{R} \times \mathbb{R}^{n} \times \mathcal{S}(n) \rightarrow \mathbb{R},
$$

where $\mathcal{S}(n)$ stands for the set of $n \times n$ real symmetric matrices, and $\Omega$ is a bounded open set in $\mathbb{R}^{n}$, typically with a sufficiently regular boundary, meaning here the uniform exterior cone condition.

We will always assume that $G(x, r, p, X)$ is continuous in $(r, p, X)$ with modulus of continuity independent of $x \in \Omega$, and is jointly measurable in all variables $(x, r, p, X)$.

We will typically require that $G$ of (1.1) satisfy the following structure conditions:

$$
\begin{gathered}
|G(x, r, p, X)-G(x, r, q, X)| \leq \gamma|p-q|, \\
\mathcal{P}^{-}(X-Y) \leq G(x, r, p, X)-G(x, r, p, Y) \leq \mathcal{P}^{+}(X-Y),
\end{gathered}
$$

for all $x \in \Omega, r \in \mathbb{R}, p, q \in \mathbb{R}^{n}$ and $X, Y \in \mathcal{S}(n)$. Here $\mathcal{P}^{ \pm}$are the Pucci extremal operators defined as

$$
\mathcal{P}^{+}(X)=-\lambda \operatorname{tr}\left(X^{+}\right)+\Lambda \operatorname{tr}\left(X^{-}\right), \quad \mathcal{P}^{-}(X)=-\Lambda \operatorname{tr}\left(X^{+}\right)+\lambda \operatorname{tr}\left(X^{-}\right),
$$

Received by the editors July $5,2000$.

2000 Mathematics Subject Classification. Primary 35J60, 35J65, 35J25, 49L25.

The first author was supported in part by NSF grants DMS-9532030, DMS-9972043 and DMS9706760.

The second author was supported by an Alexander von Humboldt Fellowship.

The third author was supported in part by NSF grant DMS-9706760. Part of this work was completed while this author was visiting the University of Cologne, supported by the TMR Network "Viscosity Solutions and their Applications".

(C)2001 American Mathematical Society 
where $\operatorname{tr}(X)$ is the trace of $X ; \lambda \leq \Lambda$, and $\gamma$ are positive constants which are fixed for all time; and given $X \in \mathcal{S}(n), X^{+}$and $X^{-}$are its positive and negative parts $\left(X=X^{+}-X^{-}\right)$. Thus (1.3) amounts to uniform ellipticity of $G$, with ellipticity constants $\lambda$ and $\Lambda$.

Sometimes we will allow $G$ to be just degenerate elliptic, meaning that

$$
G(x, r, p, X) \leq G(x, r, p, Y) \text { whenever } X-Y \quad \text { is nonnegative definite. }
$$

As for the dependence on $r$, we will assume that

$$
\left\{\begin{array}{l}
r \mapsto G(x, r, p, X) \text { is uniformly continuous, } \\
\text { uniformly for } x \in \Omega \text { and bounded }(r, p, X) .
\end{array}\right.
$$

Typically we will also request that $G$ be proper, i.e. that

$$
r \mapsto G(x, r, p, X) \quad \text { is nondecreasing. }
$$

We will frequently write (1.1) as

$$
F\left(x, u, D u, D^{2} u\right)=f(x)
$$

by setting $f(x)=-G(x, 0,0,0), F(x, r, p, X)=G(x, r, p, X)+f(x)$, so that

$$
F(x, 0,0,0) \equiv 0 \text {. }
$$

In what follows we will move freely between (1.7) and (1.1). As explained above, $f$ is just a measurable function on $\Omega$. Regarding its behavior, we will require that

$$
f \in L^{p}(\Omega), \quad p>p_{0},
$$

where $p_{0}<n$ is a constant such that the generalized maximum principle holds for $p>p_{0}$ (see [2, 6, 10]).

Clearly, $F$ of (1.7) will satisfy versions of (1.2)-(1.6) whenever $G$ of (1.1) does, and vice versa.

We recall next the definitions of strong and $L^{p}$-viscosity solutions of (1.7). We refer the reader to [2, 5] and [10] for the theory of $L^{p}$-viscosity solutions and to [1] for an overview of recent results on fully nonlinear elliptic equations.

A function $u \in W_{\text {loc }}^{2, p}(\Omega)$ is a strong solution of (1.1) if (1.1) holds pointwise a.e., i.e.

$$
G\left(x, u(x), D u(x), D^{2} u(x)\right)=0 \quad \text { for a.e. } x \in \Omega .
$$

A function $u \in C(\Omega)$ is an $L^{p}$-viscosity subsolution (respectively, supersolution) of (1.1) if for every "test function" $\varphi \in W_{\mathrm{loc}}^{2, p}(\Omega)$ and local maximum (respectively, minimum) point $\hat{x} \in \Omega$ of $u-\varphi$

$$
\begin{gathered}
\operatorname{ess} \liminf _{x \rightarrow \hat{x}} G\left(x, u(x), D \varphi(x), D^{2} \varphi(x)\right) \leq 0 \\
\text { (respectively, } \left.\quad \operatorname{ess} \limsup _{x \rightarrow \hat{x}} G\left(x, u(x), D \varphi(x), D^{2} \varphi(x)\right) \geq 0\right) .
\end{gathered}
$$

A function $u \in C(\Omega)$ is an $L^{p}$-viscosity solution of (1.1) if it is both an $L^{p}$-viscosity subsolution and an $L^{p}$-viscosity supersolution of (1.1).

In the case of linear equations

$$
-\sum_{i, j=1}^{n} a_{i j}(x) u_{x_{i} x_{j}}(x)+\sum_{j=1}^{n} b_{j}(x) u_{x_{j}}(x)+c(x) u(x)-f(x)=0
$$


a notion of so-called good solution has been proposed in [3. Namely, $u \in C(\Omega)$ is a good solution of (1.10) if there is a sequence $u^{m}$ of strong solutions of approximate problems

$$
-\sum_{i, j=1}^{n} a_{i j}^{m}(x) u_{x_{i} x_{j}}^{m}(x)+\sum_{j=1}^{n} b_{j}^{m}(x) u_{x_{j}}^{m}(x)+c^{m}(x) u^{m}(x)-f^{m}(x)=0
$$

such that $u^{m} \rightarrow u$ in $C(\Omega)$. It was proved in 8] (see also [5]) that the notions of good and $L^{p}$-viscosity solutions of (1.10) coincide, at least in the case with $b_{j} \equiv 0$, $c \equiv 0$ and $f \in L^{\infty}(\Omega)$.

We will say that the functions $G_{1}, G_{2}, \ldots, G_{m}, \ldots$ satisfy structure conditions uniformly in $m$ if (1.2), (1.3), (1.5) are satisfied uniformly in $m$ with the same fixed $\lambda, \Lambda, \gamma$, and if $\left|G_{m}(x, 0,0,0),\right| \leq g(x)$ for some $g \in L^{p}(\Omega)$.

It was hinted in [5] how to extend the notion of a good solution to fully nonlinear equations. Here we make this precise.

Definition 1.1. We say that $u \in C(\Omega)$ is a good solution of (1.1) if there exist $G_{m}$ satisfying structure conditions uniformly in $m$ and strong solutions $u_{m}$ of $G_{m}=0$ in $\Omega$, such that $u_{m} \rightarrow u$ in $C(\Omega)$ and $G_{m}$ converge to $G$ in the following sense:

$$
\begin{aligned}
& G_{m}(x, t, p, X) \rightarrow G(x, t, p, X) \\
& \quad \text { for a.e. } x \in \Omega \text { and all }(t, p, X) \in \mathbb{R} \times \mathbb{R}^{n} \times \mathcal{S}(n) .
\end{aligned}
$$

The requirement that the constants $\lambda, \Lambda, \gamma$ be fixed for all equations comes from the fact that the constant $p_{0}$ in (1.9) depends on them and on diam $(\Omega)$.

The main result of this paper shows that the notions of $L^{p}$-viscosity solution and good solution of (1.1) coincide, generalizing the results of [8] and [5] for linear equations to the general case of $f \in L^{p}(\Omega)$ and nonzero $b_{j}$ and $c$. This paper provides another tool for the analysis nonlinear elliptic partial differential equations with measurable spatial dependence. For example, using our main theorem, the results in [3] on uniqueness of good solutions translate immediately into corresponding results on uniqueness of $L^{p}$-viscosity solutions.

\section{Constructing Strong SOlutions}

We are going to construct a strong solution of the Dirichlet problem

$$
-\Delta u+G\left(x, u, D u, D^{2} u\right)=0 \quad \text { in } \Omega, \quad u=\psi \quad \text { on } \partial \Omega
$$

when $G$ is bounded. This will turn out to be an important construction in proving that an $L^{p}$-viscosity solution of (1.1) is a good solution of (1.1) as well.

Proposition 2.1. Let $G: \Omega \times \mathbb{R} \times \mathbb{R}^{n} \times \mathcal{S}(n) \rightarrow \mathbb{R}$ be measurable, bounded and satisfy (1.2), (1.4) and (1.5), let $\psi \in C(\partial \Omega)$, and let $\Omega$ satisfy uniform exterior cone condition. Then the Dirichlet problem (2.1) has a strong solution $u \in C(\bar{\Omega}) \cap$ $W_{\text {loc }}^{2, p}(\Omega)$ for every $p<\infty$.

For existence we do not require $G$ to be proper - the only essential ingredients are the boundedness and ellipticity of $G$-and in fact, we need this greater generality later. However, if $G$ satisfies (1.6), then the constructed strong solution is unique.

Proof. We will solve (2.1) by the fixed point method. To this end, for any given $v \in C(\bar{\Omega})$ we will consider the Dirichlet problem

$$
-\Delta u+G\left(x, v(x), D u, D^{2} u\right)=0 \quad \text { in } \Omega, \quad u=\psi \quad \text { on } \partial \Omega .
$$


Since the equation in (2.2) is independent of $u$, it follows from the general theory (see [4], Theorem 4.1) that (2.2) has an $L^{p}$-viscosity solution (for any finite $p$ ) in $C(\bar{\Omega})$, which we are going to denote by $T v$. By [2], Proposition 3.5, Tv is twice pointwise differentiable a.e., thus $g(x)=G\left(x, v(x), D(T v)(x), D^{2}(T v)(x)\right)$ is welldefined and $g \in L^{\infty}(\Omega)$ since $G$ is bounded. It follows that $T v$ is a pointwise a.e. - and therefore $L^{p}$-viscosity (see [10, Corollary 1.6) - solution of the Dirichlet problem

$$
-\Delta u=-g(x) \quad \text { in } \Omega, \quad u=\psi \quad \text { on } \partial \Omega .
$$

However, (2.3) clearly has a unique strong solution, which must coincide with $T v$, and it follows that $T v \in W_{\text {loc }}^{2, p}(\Omega)$ for every finite $p$. In particular, $T v$ is a unique strong solution of (2.2).

To finish the proof it is now enough to show that the map $T: C(\bar{\Omega}) \rightarrow C(\bar{\Omega})$ has a fixed point. This, however, is fairly obvious. For $R$ sufficiently large $T$ is a compact mapping from the closed ball of radius $R$ in $C(\bar{\Omega})$ to itself. Hence $T$ has a fixed point and the proof is complete.

We close this section with a direct construction of good solutions of

$$
F\left(x, u, D u, D^{2} u\right)=f(x) \quad \text { in } \Omega, \quad u=\psi \quad \text { on } \partial \Omega
$$

under the assumptions that $\psi \in C(\partial \Omega), \Omega$ satisfies uniform exterior cone condition, $F$ is measurable and satisfies (1.2), (1.3), (1.5), (1.6), (1.8), and (1.9). Under the same conditions we know that (2.4) also has an $L^{p}$-viscosity solution; see Theorem 4.1 in [4]. The construction uses Proposition 2.1, illustrating (in a simpler context) how it is applied in the following section.

We will rewrite the differential equation in (2.4) as

$$
-\lambda \Delta u+G\left(x, u, D u, D^{2} u\right)=0,
$$

where $G(x, r, p, X)=\lambda \operatorname{tr}(X)+F(x, r, p, X)-f(x)$. Clearly $G$ is degenerate elliptic, i.e. it satisfies (1.4).

Without loss of generality we may replace (2.4) by

$$
-\lambda \Delta u+G\left(x, u, D u, D^{2} u\right)=0 \quad \text { in } \Omega, \quad u=\psi \quad \text { on } \partial \Omega .
$$

For $m=1,2, \ldots$ consider truncating functions $\chi_{m}: \mathbb{R} \rightarrow \mathbb{R}$ given by

$$
\chi_{m}(r)= \begin{cases}-m & \text { for } r<-m, \\ r & \text { for } r \in[-m, m], \\ m & \text { for } r>m .\end{cases}
$$

For every $m$ we will consider an approximating Dirichlet problem

$$
-\lambda \Delta u+\chi_{m}\left(G\left(x, u, D u, D^{2} u\right)\right)=0 \quad \text { in } \Omega, \quad u=\psi \quad \text { on } \partial \Omega .
$$

Since $\chi_{m}(G(x, r, p, X))$ satisfies the conditions of Proposition 2.1 and $G$ is proper, (2.7) has a unique strong solution $u_{m} \in C(\bar{\Omega}) \cap W_{\text {loc }}^{2, p}(\Omega)$ for every $p<\infty$. The family of equations satisfies structure conditions uniformly in $m$ and so by Proposition 4.2 in 4 the $u_{m}$ are precompact in $C(\bar{\Omega})$, and therefore - passing to a subsequence if necessary - we can assume that $u_{m} \rightarrow u$ in $C(\bar{\Omega})$. The function $u \in C(\bar{\Omega})$ is a desired good solution since the approximations in (2.7) obviously converge to $G$ in the sense of (1.11). 


\section{Viscosity SOLUTIONS ARE GOOD SOLUTIONS}

Consider the Dirichlet problem

$$
F\left(x, D u, D^{2} u\right)=f(x) \quad \text { in } \Omega, \quad u=\psi \quad \text { on } \partial \Omega .
$$

We will prove that then every $L^{p}$-viscosity solution of (3.1) is a good solution. The fact that good solutions are $L^{p}$-viscosity solutions is obvious from the definition of good solutions and the general theory - convergence and stability — of $L^{p}$-viscosity solutions (see 2], Theorem 3.8).

Theorem 3.1. Let $F$ be measurable and satisfy (1.2), (1.3), (1.8), let $f$ satisfy (1.9), let $\psi \in C(\partial \Omega)$, and let $\Omega$ satisfy uniform exterior cone condition. Then every $L^{p}$-viscosity solution of (3.1) is a good solution in the sense of Definition 1.1, i.e. there is a sequence of operators $F_{m}$, independent of $u$, satisfying (1.2), (1.3) and (1.8), a sequence $f_{m} \in L^{p}(\Omega)$ and a sequence $u_{m} \in C(\bar{\Omega}) \cap W_{\text {loc }}^{2, p}(\Omega)$ of strong solutions of

$$
F_{m}\left(x, D u_{m}, D^{2} u_{m}\right)=f_{m}(x) \text { in } \Omega
$$

such that

$$
u_{m} \rightarrow u \quad \text { in } C(\bar{\Omega})
$$

$F_{m}$ converge to $F$ in the sense of (1.11):

$$
\begin{gathered}
F_{m}(x, p, X) \rightarrow F(x, p, X) \quad \text { for a.e. } x \in \Omega \\
\text { and all }(t, p, X) \in \mathbb{R} \times \mathbb{R}^{n} \times \mathcal{S}(n),
\end{gathered}
$$

and

$$
f_{m} \rightarrow f \quad \text { in } L^{p}(\Omega) \text { and a.e. in } \Omega \text {. }
$$

Observe that $F$ in (3.1) is independent of $u$. The result holds with $u$ dependence as well, assuming that $F$ is proper; the proof is the same in all essential features as it is without $u$ dependence. However, the introduction of $u$ dependence introduces additional terms which clutter up the proof and further obscure the fundamental ideas behind the proof. For these reasons we present the result without $u$ dependence.

Recall that Jensen in [8] (see also [5]) proved that $L^{n}$-viscosity solutions of linear equations (1.10) with $f \in L^{\infty}(\Omega)$ are good solutions. Our result generalizes this to general (subject to structure conditions) fully nonlinear equations and $f \in L^{p}(\Omega), p>p_{0}$.

Proof. Step 0. Fix a countable, dense in $\mathbb{R}^{n} \times \mathcal{S}(n)$ sequence $\left(p_{i}, X_{i}\right) \in \mathbb{R}^{n} \times \mathcal{S}(n)$, $i=1,2, \ldots$.

Step 1. Choose a sequence $\tilde{f}_{m} \in C(\Omega) \cap L^{\infty}(\Omega)$ such that

$$
\delta_{m}=\left\|f-\tilde{f}_{m}\right\|_{L^{p}(\Omega)} \rightarrow 0 .
$$

Consider the Dirichlet problem

$$
\mathcal{P}^{-}\left(D^{2} w\right)-\gamma|D w|=f-\tilde{f}_{m} \quad \text { in } \Omega, \quad w=0 \quad \text { on } \partial \Omega .
$$

By Corollary 3.10 in [2], (3.6) has a unique strong solution $w$, and by the maximum principle $\|w\|_{L^{\infty}(\Omega)} \leq C \delta_{m}$. Let $\bar{u}_{m}=u-w$. It follows that $\bar{u}_{m}$ is an $L^{p}$-viscosity solution of

$$
F\left(x, D \bar{u}_{m}, D^{2} \bar{u}_{m}\right) \leq \tilde{f}_{m}(x) \quad \text { in } \Omega
$$


and

$$
\left\|u-\bar{u}_{m}\right\|_{L^{\infty}(\Omega)}=\|w\|_{L^{\infty}(\Omega)} \leq C \delta_{m} .
$$

Similarly, solving

$$
\mathcal{P}^{+}\left(D^{2} w\right)+\gamma|D w|=f-\tilde{f}_{m} \quad \text { in } \Omega, \quad w=0 \quad \text { on } \partial \Omega
$$

and setting $\underline{u}_{m}=u-w$ we conclude that $\underline{u}_{m}$ is an $L^{p}$-viscosity solution of

$$
F\left(x, D \underline{u}_{m}, D^{2} \underline{u}_{m}\right) \geq \tilde{f}_{m}(x) \text { in } \Omega
$$

and

$$
\left\|u-\underline{u}_{m}\right\|_{L^{\infty}(\Omega)} \leq C \delta_{m} .
$$

Also $\bar{u}_{m}=u=\psi=\underline{u}_{m}$ on $\partial \Omega$.

Step 2. Let $\Omega_{m} \subset \subset \Omega$ be a subdomain of $\Omega$ with smooth boundary and such that

$$
x \in \Omega \backslash \Omega_{m} \Rightarrow \operatorname{dist}(x, \partial \Omega) \leq \frac{1}{m} .
$$

Next we are going to regularize $\bar{u}_{m}$ and $\underline{u}_{m}$ on $\Omega_{m}$ by means of the by-now standard process of sup-inf convolution; see [9]. An equivalent approximation procedure was used in [8] without bringing up the connection to sup-inf convolutions. This connection has been pointed out in [5], and here we will follow the approach of [5].

Recall that for a given continuous function $w: \bar{\Omega} \rightarrow \mathbb{R}$, for $\epsilon>0$ its supconvolution $w^{\epsilon}$ and its inf-convolution $w_{\epsilon}$ are defined as

$$
w^{\epsilon}(x)=\sup _{y \in \Omega}\left(\left(w(y)-\frac{1}{2 \epsilon}|x-y|^{2}\right)\right), \quad w_{\epsilon}(x)=\inf _{y \in \Omega}\left(w(y)+\frac{1}{2 \epsilon}|x-y|^{2}\right), \quad x \in \Omega .
$$

Now for $\epsilon, \delta>0$ we consider $w^{\epsilon, \delta}=\left(w^{\epsilon+\delta}\right)_{\delta}$. It is well known that if $\epsilon, \delta$ are sufficiently small, then $w^{\epsilon, \delta}$ is $C^{1,1}$ on $\Omega_{m}$, and $w^{\epsilon, \delta}$ converge to $w$ as $\epsilon, \delta \downarrow 0$; see [9]. Moreover, this approximation procedure respects viscosity subsolutions; see [5], Section 4.

First we will consider $\left(\bar{u}_{m}\right)^{\epsilon, \delta}$. Since $\tilde{f}_{m}$ are bounded, it follows from the results in [8] and [5] (in particular see Proposition 4.6 in [5]) that for every sufficiently small $\epsilon$ one can choose a suitable $\delta=\delta(\epsilon)$ so that $u_{m}^{+}=\left(\bar{u}_{m}\right)^{\epsilon, \delta} \in W^{2, \infty}\left(\overline{\Omega_{m}}\right)$ and is a strong solution of a perturbed version of (3.7), namely

$$
\bar{F}_{m}\left(x, D u_{m}^{+}(x), D^{2} u_{m}^{+}(x)\right) \leq \bar{f}_{m}(x) \quad \text { for a.e. } x \in \Omega_{m} \text {. }
$$

Here

$$
\bar{F}_{m}(x, p, X)=F\left(T_{m}^{+} x, p, X\right) \text { and } \bar{f}_{m}(x)=\tilde{f}_{m}\left(T_{m}^{+} x\right)
$$

with $T_{m}^{+} x=x+\epsilon D u_{m}^{+}$. While the equations considered in [8] and [5] were purely second order, the computations carried out there show that first order terms can be accommodated as well.

Similarly, setting $w_{\epsilon, \delta}=\left(w_{\epsilon+\delta}\right)^{\delta}$, we conclude that $u_{m}^{-}=\left(\underline{u}_{m}\right)_{\epsilon, \delta} \in W^{2, \infty}\left(\overline{\Omega_{m}}\right)$ and

$$
\underline{F}_{m}\left(x, D u_{m}^{-}(x), D^{2} u_{m}^{-}(x)\right) \geq \underline{f}_{m}(x) \text { for a.e. } x \in \Omega_{m},
$$

where now

$$
\underline{F}_{m}(x, p, X)=F\left(T_{m}^{-} x, p, X\right) \text { and } \underline{f}_{m}(x)=\tilde{f}_{m}\left(T_{m}^{-} x\right)
$$

with $T_{m}^{-} x=x-\epsilon D u_{m}^{-}$. 
Derivations of (3.12) and (3.14) use the fact that there is $\beta_{m}>0$, independent of $\epsilon$, such that

$$
D T_{m}^{ \pm} \geq \beta_{m} I \quad \text { a.e. in } \Omega_{m} .
$$

See 8 and Section 4 of [5] for details. It follows from 3.16 that $\left(T_{m}^{ \pm}\right)^{-1}$ map null sets into null sets, and therefore $\underline{F}_{m}$ and $\bar{F}_{m}$ are measurable. Moreover, by (3.16) the composition with $T_{m}^{ \pm}$is an approximate identity in $L^{p}\left(\Omega_{m}\right)$ and thereforedecreasing $\epsilon$ if necessary - we can achieve that

$$
\left\|\bar{f}_{m}-\tilde{f}_{m}\right\|_{L^{p}\left(\Omega_{m}\right)}, \quad\left\|\underline{f}_{m}-\tilde{f}_{m}\right\|_{L^{p}\left(\Omega_{m}\right)} \leq \frac{1}{m}
$$

and

$$
\int_{\Omega_{m}}\left|F\left(T_{m}^{ \pm} x, p_{i}, X_{i}\right)-F\left(x, p_{i}, X_{i}\right)\right| d x \leq \frac{1}{m} \quad \text { for } i=1,2, \ldots, m .
$$

Further, without loss of generality, we may also assume that

$$
\left\|\bar{u}_{m}-u_{m}^{+}\right\|_{L^{\infty}\left(\Omega_{m}\right)},\left\|\underline{u}_{m}-u_{m}^{-}\right\|_{L^{\infty}\left(\Omega_{m}\right)} \leq C \delta_{m} .
$$

Finally, redefining $u_{m}^{-}=u_{m}^{-}-3 C \delta_{m}$ and $u_{m}^{+}=u_{m}^{+}+3 C \delta_{m}$ we obtain that (3.12) and (3.14) still hold, while by (3.8), 3.10) and (3.19)

$$
u-5 C \delta_{m} \leq u_{m}^{-} \leq u-C \delta_{m}, \quad u+C \delta_{m} \leq u_{m}^{+} \leq u+5 C \delta_{m} \quad \text { on } \overline{\Omega_{m}} .
$$

Step 3. Before going any further with the construction of approximating equations, here we will establish some limiting properties of the approximations constructed in Step 2, which will be needed later in Step 6. From (3.18), for every $\left(p_{i}, X_{i}\right)$ and $\Omega^{\prime \prime} \subset \subset \Omega$

$$
\int_{\Omega^{\prime \prime}}\left|F\left(T_{m}^{ \pm} x, p_{i}, X_{i}\right)-F\left(x, p_{i}, X_{i}\right)\right| d x \rightarrow 0 \quad \text { as } m \rightarrow \infty .
$$

By a diagonal argument we construct a subsequence $m_{k}$ and a null set $\mathcal{N} \subset \Omega$ such that

$$
x \in \Omega \backslash \mathcal{N} \Rightarrow F\left(T_{m_{k}}^{ \pm} x, p_{i}, X_{i}\right) \rightarrow F\left(x, p_{i}, X_{i}\right) \quad \text { as } k \rightarrow \infty, \text { for all } i,
$$

and using structure conditions (1.2) and (1.3) we can generalize this to

$$
\begin{aligned}
x \in \Omega \backslash \mathcal{N} \Rightarrow F\left(T_{m_{k}}^{ \pm} x, p, X\right) \rightarrow & F(x, p, X) \quad \text { as } k \rightarrow \infty, \\
& \text { for all }(p, X) \in \mathbb{R}^{n} \times \mathcal{S}(n) .
\end{aligned}
$$

Step 4. Next choose a constant $M_{m} \geq m$ so that for a.e. $x \in \overline{\Omega_{m}}$

$$
\begin{aligned}
& \left|\bar{F}_{m}\left(x, D u_{m}^{+}(x), D^{2} u_{m}^{+}(x)\right)+\lambda \Delta u_{m}^{+}(x)\right|, \\
& \left|\underline{F}_{m}\left(x, D u_{m}^{-}, D^{2} u_{m}^{-}\right)+\lambda \Delta u_{m}^{-}(x)\right| \leq M_{m} .
\end{aligned}
$$

Writing

$$
\bar{G}_{m}(x, p, X)=-\lambda \operatorname{tr}(X)+\chi_{M_{m}}\left(\bar{F}_{m}(x, p, X)+\lambda \operatorname{tr}(X)\right),
$$

it follows from (3.12) and (3.22) that

$$
\bar{G}_{m}\left(x, D u_{m}^{+}(x), D^{2} u_{m}^{+}(x)\right) \leq \bar{f}_{m}(x) \quad \text { for a.e. } x \in \Omega_{m} \text {. }
$$

Similarly,

$$
\underline{G}_{m}\left(x, D u_{m}^{-}(x), D^{2} u_{m}^{-}(x)\right) \geq \underline{f}_{m}(x) \quad \text { for a.e. } x \in \Omega_{m},
$$


where now

$$
\underline{G}_{m}(x, p, X)=-\lambda \operatorname{tr}(X)+\chi_{M_{m}}\left(\underline{F}_{m}(x, p, X)+\lambda \operatorname{tr}(X)\right) .
$$

The next step of the proof is similar to the proof of Theorem 3.30 in [8]. Let $\mu: \overline{\Omega_{m}} \times \mathbb{R} \rightarrow[0,1]$ be a continuous function such that

$$
\mu(x, t)=0 \quad \text { if } u_{m}^{+}(x) \leq t, \quad \mu(x, t)=1 \quad \text { if } u_{m}^{-}(x) \geq t .
$$

This can be done due to (3.20).

Define

$$
\begin{gathered}
H_{m}(x, r, p, X)=\mu(x, r) \bar{G}_{m}(x, p, X)+(1-\mu(x, r)) \underline{G}_{m}(x, p, X) \\
=-\lambda \operatorname{tr}(X)+\mu(x, r) \chi_{M_{m}}\left(\bar{F}_{m}(x, p, X)+\lambda \operatorname{tr}(X)\right) \\
+(1-\mu(x, r)) \chi_{M_{m}}\left(\underline{F}_{m}(x, p, X)+\lambda \operatorname{tr}(X)\right), \\
h_{m}(x, r)=\mu(x, r) \bar{f}_{m}(x)+(1-\mu(x, r)) \underline{f}_{m}(x)
\end{gathered}
$$

and consider the Dirichlet problem

$$
H_{m}\left(x, v, D v, D^{2} v\right)=h_{m}(x, v) \quad \text { in } \Omega_{m}, \quad v=u \quad \text { on } \partial \Omega_{m} .
$$

All assumptions of Proposition 2.1 are satisfied and thus (3.30) has a strong solution $v_{m} \in C\left(\overline{\Omega_{m}}\right) \cap W_{\text {loc }}^{2, p}\left(\Omega_{m}\right)$. Now we define

$$
G_{m}(x, p, X)=H_{m}\left(x, v_{m}(x), p, X\right), \quad g_{m}(x)=h_{m}\left(x, v_{m}(x)\right),
$$

so that $v_{m}$ solves

$$
G_{m}\left(x, D v_{m}(x), D^{2} v_{m}(x)\right)=g_{m}(x) \quad \text { for a.e. } x \in \Omega_{m}, \quad v_{m}=u \quad \text { on } \partial \Omega_{m} .
$$

Observe that by construction

$$
\left\|g_{m}\right\|_{L^{\infty}\left(\Omega_{m}\right)} \leq\left\|\tilde{f}_{m}\right\|_{L^{\infty}(\Omega)}, \quad\left|\lambda \operatorname{tr}(X)+G_{m}(x, p, X)\right| \leq M_{m} .
$$

Moreover, from (3.17) and (3.29)

$$
\left\|g_{m}-\tilde{f}_{m}\right\|_{L^{p}\left(\Omega_{m}\right)} \leq\left\|\bar{f}_{m}-\tilde{f}_{m}\right\|_{L^{p}\left(\Omega_{m}\right)}+\left\|\underline{f}_{m}-\tilde{f}_{m}\right\|_{L^{p}\left(\Omega_{m}\right)} \leq \frac{2}{m} .
$$

Next we claim that

$$
v_{m} \leq u_{m}^{+}+8 C \delta_{m} \text { on } \overline{\Omega_{m}} .
$$

To show (3.35) consider $\Omega_{m}^{+}=\left\{x \in \Omega_{m}: v_{m}(x)>u_{m}^{+}(x)\right\}$. Then $\mu\left(x, v_{m}(x)\right) \equiv 0$ on $\Omega_{m}^{+}$and therefore $g_{m}(x)=\underline{f}_{m}(x)$ and $G_{m}(x, p, X)=\underline{G}_{m}(x, p, X)$ for $x \in \Omega_{m}^{+}$. It follows that $v_{m}$ is a strong solution of $\underline{G}_{m}=\underline{f}_{m}$ on $\Omega_{m}^{+}$, and since by $3.25 u_{m}^{-}$ is a supersolution of the same equation, by the minimum principle

$$
\begin{aligned}
& \inf _{\Omega_{m}^{+}}\left(u_{m}^{-}-v_{m}\right) \geq \inf _{\partial \Omega_{m}^{+}}\left(u_{m}^{-}-v_{m}\right) \\
& \quad \geq \min \left(\inf _{\partial \Omega_{m}^{+} \backslash \partial \Omega_{m}}\left(u_{m}^{-}-u_{m}^{+}\right), \inf _{\partial \Omega_{m}}\left(u_{m}^{-}-u\right)\right) \geq-10 C \delta_{m}
\end{aligned}
$$

by (3.20), and using (3.20) again yields (3.35).

A symmetric argument shows that

$$
v_{m} \geq u_{m}^{-}-8 C \delta_{m} \quad \text { on } \overline{\Omega_{m}} .
$$

Putting (3.35), (3.36) and (3.20) together gives

$$
u-13 C \delta_{m} \leq v_{m} \leq u+13 C \delta_{m} \text { on } \overline{\Omega_{m}} .
$$


Step 5. We will extend $g_{m}$ and $G_{m}$ to the whole $\Omega$ according to

$$
\begin{aligned}
f_{m}(x) & = \begin{cases}g_{m}(x) & \text { for } x \in \Omega_{m}, \\
0 & \text { for } x \in \Omega \backslash \Omega_{m},\end{cases} \\
F_{m}(x, p, X) & = \begin{cases}G_{m}(x, p, X) & \text { for } x \in \Omega_{m}, \\
-\lambda \operatorname{tr}(X) & \text { for } x \in \Omega \backslash \Omega_{m} .\end{cases}
\end{aligned}
$$

Recalling (3.33), by Proposition 2.1 the Dirichlet problem

$$
F_{m}\left(x, D w, D^{2} w\right)=f_{m} \quad \text { in } \Omega, \quad u=\psi \quad \text { on } \partial \Omega
$$

has a unique strong solution $u_{m} \in C(\bar{\Omega}) \cap W_{\text {loc }}^{2, p}(\Omega)$ for every $p<\infty$. Moreover, by Remark 4.3 in 4 there exists a modulus of continuity $\rho$ determined only by $\lambda, \Lambda, n, p, \gamma,\left\|f_{m}\right\|_{L^{p}(\Omega)}$, the modulus of continuity of $\psi$, diam $(\Omega)$ and the parameters of the cone condition for $\Omega$ (and therefore independent of $m$ ) such that

$$
\left|u_{m}(x)-\psi(y)\right| \leq \rho(|x-y|) \quad \text { for } x \in \Omega, y \in \partial \Omega .
$$

Step 6. Denoting by $\rho_{u}$ the modulus of continuity of $u$ on $\bar{\Omega}$ we conclude from (3.11) and (3.40) that

$$
\left|u_{m}(x)-u(x)\right| \leq \rho\left(\frac{1}{m}\right)+\rho_{u}\left(\frac{1}{m}\right) \quad \text { for } x \in \Omega \backslash \Omega_{m} .
$$

Since both $u_{m}$ and $v_{m}$ solve the same equation $G_{m}=g_{m}$ in $\Omega_{m}$ while $v_{m}=u$ on $\partial \Omega_{m}$, by the maximum principle and (3.41)

$$
\sup _{x \in \Omega_{m}}\left|u_{m}-v_{m}\right| \leq \sup _{x \in \partial \Omega_{m}}\left|u_{m}-u\right| \leq \rho\left(\frac{1}{m}\right)+\rho_{u}\left(\frac{1}{m}\right),
$$

and this, together with (3.37), yields

$$
\sup _{x \in \Omega_{m}}\left|u_{m}-u\right| \leq \rho\left(\frac{1}{m}\right)+\rho_{u}\left(\frac{1}{m}\right)+13 C \delta_{m} .
$$

Using (3.41) again we conclude that

$$
\sup _{x \in \Omega}\left|u_{m}-u\right| \leq \rho\left(\frac{1}{m}\right)+\rho_{u}\left(\frac{1}{m}\right)+13 C \delta_{m},
$$

and therefore (3.3) follows.

From (3.34)

$$
\begin{aligned}
\left\|f_{m}-f\right\|_{L^{p}(\Omega)} & \leq\|f\|_{L^{p}\left(\Omega \backslash \Omega_{m}\right)}+\left\|g_{m}-\tilde{f}_{m}\right\|_{L^{p}\left(\Omega_{m}\right)}+\left\|f-\tilde{f}_{m}\right\|_{L^{p}(\Omega)} \\
& \leq\|f\|_{L^{p}\left(\Omega \backslash \Omega_{m}\right)}+\frac{2}{m}+\delta_{m},
\end{aligned}
$$

which, together with (3.11), establishes (3.5) along a subsequence.

We will finish the proof by showing that the convergence in (3.4) holds along a subsequence $m_{k}$ constructed in Step 3; recall (3.21). To this end, we will show that $F_{m_{k}}$ converge to $F$ pointwise a.e. in the sense that

$$
\begin{array}{r}
x \in \Omega \backslash \mathcal{N} \Rightarrow F_{m_{k}}(x, p, X) \rightarrow F(x, p, X) \text { as } k \rightarrow \infty, \\
\text { for all }(p, X) \in \mathbb{R}^{n} \times \mathcal{S}(n) .
\end{array}
$$

However, $F_{m}$ is just a convex combination of truncations of $\bar{F}_{m}$ and $\underline{F}_{m}$ defined in (3.13) and (3.15) (recall (3.38), (3.31), (3.28), (3.26) and (3.23)), and hence (3.43) follows easily from (3.21). 


\section{REFERENCES}

1. L. A. Caffarelli and X. Cabré, Fully nonlinear elliptic equations, American Mathematical Society, Providence, 1995. MR 96h:35046

2. L. Caffarelli, M. G. Crandall, M. Kocan and A. Swiech, On viscosity solutions of fully nonlinear equations with measurable ingredients, Comm. Pure Appl. Math. 49 (1996), 365-397. MR 97a:35051

3. M. C. Cerrutti, L. Escauriaza and E. B. Fabes, Uniqueness in the Dirichlet problem for some elliptic operators with discontinuous coefficients, Ann. Mat. Pura Appl. 163 (1993), 161-180. MR 94d:35034

4. M. G. Crandall, M. Kocan, P. L. Lions and A. Swiech, Existence results for boundary problems for uniformly elliptic and parabolic fully nonlinear equations, Electronic J. Differential Equations 24 (1999), 1-20. MR 2000f:35052

5. M. G. Crandall, M. Kocan, P. Soravia and A. Swiech, On the equivalence of various weak notions of solutions of elliptic PDE's with measurable ingredients, in Progress in elliptic and parabolic partial differential equations, (A. Alvino et al. eds.), Pitman Research Notes in Math., vol. 50, 1996, 136-162. MR 97m:35033

6. L. Escauriaza, $W^{2, n}$ a priori estimates for solutions to fully non-linear equations, Indiana Univ. Math. J. 42 (1993), 413-423. MR 94h:35022

7. D. Gilbarg and N.S. Trudinger, Elliptic partial differential equations of second order, 2nd edition, Springer-Verlag, Berlin, Heidelberg, New York, 1983. MR 86c:35035

8. R. Jensen, Uniformly elliptic PDEs with bounded, measurable coefficients, J. Fourier Anal. Appl. 2 (1996), 237-259. MR 97b:35032

9. J. M. Lasry and P. L. Lions, A remark on regularization in Hilbert spaces, Israel J. Math. $\mathbf{5 5}$ (1986), 257-266. MR 88b:41020

10. A. Swiech, $W^{1, p}$-interior estimates for solutions of fully nonlinear, uniformly elliptic equations, Adv. Differential Equat. 2 (1997), 1005-1027. MR 99a:35098

Department of Mathematical and Computer Sciences, Loyola University, Chicago, ILLINOIS 60626

E-mail address: rrj@math.luc.edu

Department of Mathemetics, University of Cologne, Cologne 50923, Germany

Current address: Maple Partners Bankhaus, Feuerbachstr. 26-32, 60325 Frankfurt, Germany

School of Mathematics, Georgia Institute of Technology, Atlanta, Georgia 30332

E-mail address: swiech@math.gatech.edu 